\title{
Gut Microbiota Features in Young Children With Autism Spectrum Disorders
}

\section{OPEN ACCESS}

Edited by:

Sunny Hei Wong,

The Chinese University of Hong Kong,

China

Reviewed by:

Maria Jose Gosalbes,

Centro de Investigación Biomédica en

Red (CIBER), Spain

Aymé Spor,

INRA UMR1347 Agroécologie, France

${ }^{*}$ Correspondence:

Lorena Coretti

lorena.coretti@tiscali.it

Roberto Berni Canani berni@unina.it

Francesca Lembo

frlembo@unina.it

Specialty section This article was submitted to Microbial Symbioses, a section of the journal

Frontiers in Microbiology

Received: 02 August 2018 Accepted: 04 December 2018 Published: 19 December 2018

Citation:

Coretti L, Paparo L, Riccio MP, Amato $F$, Cuomo M, Natale $A$, Borrelli L, Corrado G, De Caro C,

Comegna M, Buommino E,

Castaldo G, Bravaccio C, Chiariotti L,

Berni Canani R and Lembo F (2018)

Gut Microbiota Features in Young

Children With Autism Spectrum

Disorders. Front. Microbiol. 9:3146.

doi: 10.3389/fmicb.2018.03146
Lorena Coretti1,2,3*, Lorella Paparo ${ }^{4}$, Maria Pia Riccio ${ }^{4}$, Felice Amato ${ }^{1,5}$, Mariella Cuomo ${ }^{1}$, Alessandro Natale ${ }^{1}$, Luca Borrelli3,6, Giusi Corrado ${ }^{4}$, Carmen De Caro ${ }^{7}$, Marika Comegna ${ }^{1,5}$, Elisabetta Buommino ${ }^{3,7}$, Giuseppe Castaldo ${ }^{1,5}$, Carmela Bravaccio ${ }^{4}$, Lorenzo Chiariotti ${ }^{1,3,8}$, Roberto Berni Canani, ${ }^{3,4,5,9 *}$ and Francesca Lembo ${ }^{3,7 *}$

${ }^{1}$ Department of Molecular Medicine and Medical Biotechnology, University of Naples Federico II, Naples, Italy, ${ }^{2}$ Department of Physiology and Biochemistry, Faculty of Medicine and Surgery, University of Malta, Msida, Malta, ${ }^{3}$ Task Force on Microbiome Studies, University of Naples Federico II, Naples, Italy, ${ }^{4}$ Department of Translational Medical Science - Pediatric Section, University of Naples Federico II, Naples, Italy, ${ }^{5}$ CEINGE Advanced Biotechnologies, University of Naples Federico II, Naples, Italy, ${ }^{6}$ Department of Veterinary Medicine and Animal Productions, University of Naples Federico II, Naples, Italy, ${ }^{7}$ Department of Pharmacy, University of Naples Federico II, Naples, Italy, ${ }^{8}$ Istituto di Endocrinologia ed Oncologia Sperimantale, Naples, Italy, ${ }^{9}$ European Laboratory for the Investigation of Food-Induced Diseases, University of Naples Federico II, Naples, Italy

Proliferation and/or depletion of clusters of specific bacteria regulate intestinal functions and may interfere with neuro-immune communication and behavior in patients with autism spectrum disorder (ASD). Consistently, qualitative and quantitative alteration of bacterial metabolites may functionally affect ASD pathophysiology. Up to date, age-restricted cohort studies, that may potentially help to identify specific microbial signatures in ASD, are lacking. We investigated the gut microbiota (GM) structure and fecal short chain fatty acids (SCFAs) levels in a cohort of young children (2-4 years of age) with ASD, with respect to age-matched neurotypical healthy controls. Strong increase of Bacteroidetes and Proteobacteria and decrease of Actinobacteria was observed in these patients. Among the 91 OTUs whose relative abundance was altered in ASD patients, we observed a striking depletion of Bifidobacterium longum, one of the dominant bacteria in infant GM and, conversely, an increase of Faecalibacterium prausnitzii, a late colonizer of healthy human gut and a major butyrate producer. High levels of $F$. prausnitzii were associated to increase of fecal butyrate levels within normal range, and over representation of KEGG functions related to butyrate production in ASD patients. Here we report unbalance of GM structure with a shift in colonization by gut beneficial bacterial species in ASD patients as off early childhood.

Keywords: gut microbiome, ASD, short chain fatty acids, Faecalibacterium prausnitzii, Bifidobacterium longum, butyrate, propionate

\section{INTRODUCTION}

Autism spectrum disorders (ASD) are a group of severe neurodevelopmental conditions characterized by stereotypic behavior with defective communication and social interaction deriving from a combination of genetic and environmental factors (Hallmayer et al., 2011). The influence of early life alteration in GM and its metabolites on the development of ASD symptoms is emerging. 
In animal models with ASD behavioral traits, GM dysbiosis was correlated to behavioral alterations, gastrointestinal tract abnormalities and immunologic alterations (Hsiao et al., 2013; Coretti et al., 2017), mimicking clinical features reported in ASD patients (de Magistris et al., 2010; Estes and McAllister, 2015). Several human pre-clinical studies have described abnormal gut bacteria in children with ASD. An unbalance in Bacteroidetes and Firmicutes phyla have been described with a decrease of Bacteriodetes/Firmicutes ratio in fecal samples of autistic children (Tomova et al., 2015). Higher abundance of Clostridiun, Sutterella, Lactobacillus, Desulfovibrio genera, and Bacteroides vulgatus was found in ASD patients with respect to neurotypical subjects (Song et al., 2004; Parracho et al., 2005; Finegold et al., 2010; Williams et al., 2011; De Angelis et al., 2013). Conversely, in some studies, Bifidobacterium, Prevotella, and Akkermansia muciniphila were found reduced in GM of ASD children (Finegold et al., 2010; Wang et al., 2011; Kang et al., 2013). More recently, reduction of Bacteroidetes in ASD young subjects and, at genus level, increase of Collinsella, Corynebacterium, Dorea, and Lactobacillus together with significant reduction of Alistipes, Bilophila, Dialister, Parabacteroides, and Veillonella have been reported (Strati et al., 2017). Inconsistently with previous studies, the ratio of Bacteroidetes/Firmicutes was significantly higher in Chinese children with ASD probably due to different living environment and eating habits (Zhang et al., 2018). Along with GM composition, also SCFAs levels, which are modulated by functional gut microbes, showed changes both in human patients and mouse models of ASD, possibly contributing to ASD symptoms. Butyric, propionic, acetic, valeric acids were found increased in ASD patients compared to controls and decreased in association with probiotic use (Wang et al., 2012). Abnormal levels of SCFAs in the systemic circulation may cause metabolic and neurological effects relevant to ASD (Thomas et al., 2012; Frye et al., 2016). Findings in animal models also supported this hypothesis (MacFabe et al., 2007; de Theije et al., 2014).

Several attempts have been made in elucidating GM features and to predict GM related activities in ASD, unfortunately heterogeneity in age, enrollment criteria, and methods used for GM analysis limit our knowledge in this area. In this study, we comparatively evaluated the GM composition and fecal levels of short chain fatty acids (SCFAs) in young ASD children, with a very restricted range of age, next ending from 2 to 4 years, and in age matched healthy controls.

\section{MATERIALS AND METHODS}

\section{Study Subjects}

ASD patients (both sexes, age 2-4 years), consecutively observed at a tertiary Center for Pediatric Neuropsychiatry, were evaluated for the study.

Exclusion criteria were: ASD secondary to genetic syndromes; concomitant other neurological diseases: obesity; genetic and metabolic syndromes; immunodeficiencies; chronic diseases of the GI or respiratory tract; congenital cardiac defects; hepatic diseases; allergic diseases; food intolerances; use of antibiotics, pre-/pro- or synbiotics in the previous 4 weeks.
Patients recruitment occurred in 8 months. Twenty-five children, with suspected diagnosis of ASD, were evaluated. All children therefore carried out a full anamnestic and clinical evaluation including genetic and metabolic evaluation, eye counseling, Auditory Brainstem Response (ABR), Magnetic Resonance Imaging (MRI), electroencephalography (EEG). Children were evaluated by the pediatric neuropsychiatrist and underwent to the clinical protocol for evaluation of ASD; 20 out of 25 received a final diagnosis of ASD (first diagnosis) whereas five children did not have diagnostic confirmation or had other concurrent medical conditions (brain abnormalities, epilepsy, other genetic or metabolic diseases) and were excluded from the study. Nine out of 20 children were excluded from the study because of a recent antibiotic treatment or because of food selectivity, with dietary habits significantly different from that of the healthy controls. A total of 11 ASD patients (ASD) were recruited in this study. During the same study period 14 age matched healthy controls (HCs) visiting our Center because minor surgical procedures were enrolled (Table 1).

In all patients the diagnosis of ASD was made according to the Diagnostic and Statistical Manual of Mental Disorders, Fifth edition (DSM-5; American Psychiatric Association, 2013). In order to validate the ASD diagnosis according to Italian Guideline, all patients underwent to the Autism Diagnostic Observation Schedule - 2 (ADOS 2; Lord et al., 2012) and Autism Diagnostic Interview, Revised Version (ADI-R; Rutter et al., 2005) was administered to parents; moreover, the Griffiths Mental Development Scales (Gagliano et al., 2014), Vineland Adaptive Behavior Scales (VABS; Sparrow et al., 2003) and Childhood Autism Rating Scale (CARS; Schopler et al., 1988) were administered to better characterize the patients.

According to DSM-5 ASD criteria includes three levels of severity: Level 1 ("Requiring support"), Level 2 ("Requiring substantial support"), and Level 3 ("Requiring very substantial support"). The classification is split across two areas, Social Communication (SC) and Restricted and Repetitive Behaviors (RRB), mirroring core ASD symptoms. The notion of "level

TABLE 1 | Descriptive data of study participants.

\begin{tabular}{lcc}
\hline & ASD & HCs \\
\hline Subjects $(n)$ & 11 & 14 \\
Age (months) & $35 \pm 5.7$ & $35 \pm 8.4$ \\
Gender $(n)$ & & \\
$\quad$ Male & $81.8 \%(9)$ & $57.1 \%(8)$ \\
Female & $18.2 \%(2)$ & $42.9 \%(6)$ \\
Gl disorders $(n)$ & 2 & 0 \\
DSM-5 $(n)$ & 1 & - \\
Level-1 & 5 & - \\
Level-2 & 5 & - \\
Level-3 & $16 \pm 5.7$ & \\
ADOS (Total score) & &
\end{tabular}

ASD, autistic patients; HCs, neurotypical healthy controls; DSM-5 levels, Level 1 ("Requiring support"), Level 2 ("Requiring substantial support"), and Level 3 ("Requiring very substantial support"); ADOS, Autism Diagnostic Observation Schedules - total score. Age and ADOS scores are reported as mean $\pm S D$. 
of support" is considered as severity of impairment, the environmental modifications required for managing day-to-day life (Kats et al., 2013). ADOS total-scores overcame the cut-off for the presence of Autism in all the evaluated subjects. Based on neuropsychiatric assessment, according to DSM-5 severity-levels, 5 patients obtained scores indicating a requiring very substantial support, 5 had a requiring substantial support, and 1 showed requiring support (Table $\mathbf{1}$ ).

In all study subjects we evaluated dietary habits, with the use of a 3 days diary and results evaluated with a specific software (WinFood, Medimatica Srl Colonnella (TE), Italy), and the possible presence of functional gastrointestinal disorders using a validated questionnaire (Rome III Criteria questionnaire, Italian Version) (Walker et al., 2006). All the enrolled children were from an urban area and presented similar dietary habits.

For all study subjects a stool samples $(3 \mathrm{~g})$ was collected in sterile vials and immediately frozen at $-80^{\circ} \mathrm{C}$.

The study was approved by the Ethics Committee of the University of Naples "Federico II" and all enrolled tutors give written informed consent in accordance with the sampling protocol approved by the local ethical committee (No. 312/17). Written informed consent was obtained from the parents of the participants in this study.

\section{V3-V4 16S rRNA Gene Sequencing and Data Analysis}

Bacterial genomic DNA was extracted from frozen fecal samples using the QIAamp DNA Stool Mini Kit (Qiagen) according to manufacturer's instructions. Extracted DNAs were checked for quality and quantity by spectrophotometric measurements with NanoDrop (ThermoFisher Scientific Inc) and stored at $-20^{\circ} \mathrm{C}$ until processed for amplification. Sequencing samples were prepared according to the protocol 16S Metagenomic Sequencing Library Preparation for Illumina Miseq System with some modifications as previously described (Coretti et al., 2017). The V3-V4 regions of the 16S rDNA gene were firstly amplified starting from $200 \mathrm{ng}$ genomic DNA with Fast Start High Fidelity PCR System (Roche Applied Science) and subsequently indexed with Nextera XT Index Kit (Illumina) in 10 cycles of PCR using KAPA HiFi HotStart System according to Illumina guidelines. After each PCR step, amplicons were purified with Agencourt AMPure XP beads (Beckman Coulter Inc.). Then, library sizes and concentrations of barcoded amplicons were assessed using Bioanalyzer DNA 1000 chip (Agilent technologies) and Qubit dsDNA BR assay kit (Invitrogen), respectively. Normalized libraries were pooled, denatured with $\mathrm{NaOH}$, diluted to $10 \mathrm{pM}$ and combined with 25\% (v/v) denatured 10pM PhiX, according to Illumina guidelines. Sequencing run was performed on an Illumina Miseq system using v3 reagents for $2 \times 281$ cycles.

V3-V4 16S rDNA FASTQ paired-end reads were quality filtered and assembled using PEAR (Zhang et al., 2014), retaining only those sequences showing average PHRED score $\geq 30$, read length between 400 and 500 bp and overlapping regions between mate-pair end of at least 40 nucleotides. Passing filter sequences were processed with PRINSEQ in order to obtain FASTA and quality files for further analyses (Schmieder and Edwards, 2011).
Metagenomic analyses on the resulting data were conducted using Quantitative Insights Into Microbial Ecology (QIIME, version 1.9.1) (Caporaso et al., 2010). 16S rRNA gene sequencing reads were collapsed to operational taxonomic units (OTUs) using closed reference-based OTU picking method against Greengenes $16 \mathrm{~S}$ gene database (GG, may 2013 version) (DeSantis et al., 2006) at $97 \%$ of sequences similarity; picked OTUs were classified at different taxonomic levels with the GG database. Species and Clostridium cluster classification was performed using SPINGO version 1.3 with default parameters on a representative sequence of each OTU (Allard et al., 2015). To avoid sample size biases in subsequent analyses, a sequence rarefaction procedure was applied using a maximum depth of 45,038 sequences/sample.

To assess sampling depth coverage and species heterogeneity in each sample, alpha diversity metrics were employed on rarefied OTU table using Good's coverage, Observed species and Shannon's diversity index. A two-sample permutation t-test, using 999 Monte Carlo permutations to compute $p$-value, was performed to compare the alpha diversities between sample groups. Diversity among sample communities (beta diversity) was assessed by calculating weighted and unweighted Unifrac distance matrices and then represented by two dimensional principal coordinates analysis (PCoA) plot. Statistical significance of beta diversities was assessed on weighted and unweighted UniFrac distances matrixes using ANOSIM and ADONIS methods with 999 permutations and a two-sided Student's two-sample $t$-test. The Microbiome Regression-based Kernel Association Test (MiRKAT) (Zhao et al., 2015), a kernel-based regression method, was used to evaluate the effect of confounders by using a kernel metric constructed from weighted and unweighted UniFrac distances and adjusting for the small-sample size of the covariates. The significant associations were assessed using 9,999 permutations to verify the asymptotic p-value approximations.

Statistical differences in OTUs frequencies between sample groups at different taxonomic levels were assessed using nonparametric Kruskal-Wallis test taking into account False Discovery Rate (FDR) corrected $p$-values. Next, two analyses were applied on OTU tables generated by QIIME to identify key OTUs that discriminate ASD and HCs groups: Metastats comparison using the online interfaces (White et al., 2009) and LDA Effect Size analysis (LEfSe) (Segata et al., 2011). Only those OTUs reported by both methods to be significantly different between the two groups ( $p<0.05$ for Metastats, LDA $>2$ and $p<0.05$ for LEfSe) have been considered as key discriminatory OTUs.

The Phylogenetic Investigation of Communities by Reconstruction of Unobserved States (PICRUSt) was employed to predict the functional profile of the microbial communities in ASD and HC samples (Langille et al., 2013). In particular, the rarefied OTU table produced within QIIME was first corrected for multiple 16S rRNA gene copy number by using the normalize_by_copy_number.py script, then the obtained normalized OTU table was used as input in the predict_metagenomes.py script obtaining the Kyoto encyclopedia of genes and genomes (KEGG) ortholog (KOs) 
predictions for each sample (Kanehisa et al., 2016). KOs abundances of selected functions were compared between groups by using nonparametric Kruskal-Wallis test taking into account FDR corrected $p$-values.

Microbial interactions were investigated by generating the Spearman co-occurrence network on the basis of the relative abundances of key OTUs. The network was generated using the CoNet plugin (Faust and Raes, 2016) for Cytoscape (3.7.0, Shannon et al., 2003) by applying the following parameters: nonparametric Spearman correlation coefficients with a minimal cut-off threshold of $0.5(P<0.05$, FDR corrected), null distribution generated by 1000 permutations with renormalization, and 1000 iterations for bootstraps. Network was built taking in consideration the ADOS scores, and the fecal butyrate concentrations. Autism and butyrate's adjacent edges and connected nodes are reported as sub-networks.

\section{Droplet Digital PCR (ddPCR)}

Absolute quantification of bacteria was examined by ddPCR using a DNA binding dye (EvaGreen) according to the manufacturer's instructions. Species-specific primers for F. prausnitzii (Pfra-f: GATGGCCTCGCGTCCGATTAG and Pfra-r: CCGAAGACCTTCTTCCTCC) were designed by Chen et al. (2011). Total bacteria load in each sample was assessed using universal primers targeting the $\mathrm{V} 7$ region of the 16S rRNA gene (1048f: GTGSTGCAYGGYYGTCGTCA, 1194r: ACGTCRTCCMCNCCTTCCTC) (Raveh-Sadka et al., 2015). The ddPCR reaction mixture consisting of $10 \mu \mathrm{l}$ QX200 EvaGreen ddPCR Supermix, $100 \mathrm{nM}$ primers, and $0.5 \mathrm{ng}$ of DNA extracted from fecal samples was mixed with $70 \mu \mathrm{l}$ of droplet generator oil to create droplets following the manufacture's instructions. The following thermocycling parameters were used to PCR amplify the droplets generated from each sample: one denaturation cycle at $95^{\circ} \mathrm{C}$ for $10 \mathrm{~min}$, 40 cycles composed of $95^{\circ} \mathrm{C}$ for $30 \mathrm{~s}, 61^{\circ} \mathrm{C}$ for $30 \mathrm{~s}$, and $72^{\circ} \mathrm{C}$ for $30 \mathrm{~s}$, followed by a stabilization cycle at $98^{\circ} \mathrm{C}$ for $10 \mathrm{~min}$. All ramp rates were at $2.5^{\circ} \mathrm{C} / \mathrm{s}$. The copies $/ \mu \mathrm{l}$ output from QuantaSoft software was used to calculate the number of bacterial cells, assuming four copies for bacteria (Hospodsky et al., 2012), normalized by grams of fecal mass used for each gDNA extraction reaction (Raveh-Sadka et al., 2015).

\section{Determination of Fecal Butyrate and Propionate Concentration}

Frozen feces weighing $1 \mathrm{~g}$ were diluted with saline, vortexed and centrifuged at 13,000 r.p.m. for $10 \mathrm{~min}$ in $2 \mathrm{ml}$ tubes. The supernatants were filtered $(0.45 \mu \mathrm{m})$ and used as the fecal extracts, which were stored at $-20^{\circ} \mathrm{C}$ until analysis. To determine fecal butyrate and propionate concentration, frozen fecal extracts were acidified with $20 \mu \mathrm{l} 85 \%$ phosphoric acid and $0.5 \mathrm{ml}$ ethyl acetate, mixed, centrifuged at 14,000 r.p.m. for $1 \mathrm{~h}$ and extracted in duplicate. A quantity of the pooled extract containing the acidified butyrate was transferred into a $2 \mathrm{ml}$ glass vial and loaded onto an Agilent Technologies (Santa
Clara, CA, United States) 7890 gas chromatograph (GC) system with automatic loader/injector. The GC column was an Agilent J\&W DB-FFAP (Agilent Technologies) with the length $30 \mathrm{~m}$, internal diameter $0.25 \mathrm{~mm}$ and film thickness $0.25 \mu \mathrm{m}$. The GC was programmed to achieve the following run parameters: initial temperature $90^{\circ} \mathrm{C}$, hold $0.5 \mathrm{~min}$, ramp $20^{\circ} \mathrm{C} \mathrm{min} \mathrm{m}^{-1}$, final temperature $190^{\circ} \mathrm{C}$, total run time $8.0 \mathrm{~min}$, gas flow $7.7 \mathrm{ml} \mathrm{min}{ }^{-1}$ split less to maintain 3.26 p.s.i. column head pressure, septum purge $2.0 \mathrm{ml} \mathrm{min} \mathrm{m}^{-1}$. Detection was achieved using a flame ionization detector. Peaks were identified using a mixed external standard and quantified by peak height/internal standard ratio.

\section{Statistical Analysis}

ddPCR assay and fecal butyrate and propionate measurement results in ASD and HCs samples were compared using two tailed Student's $t$-test assuming equal variance. In this study results were considered statistically significant at $p$-value $<0.05$. Significant differences were indicated in figures by $* p<0.05$, ${ }^{* *} p<0.01,{ }^{* * *} p<0.001$. ANOSIM, ADONIS and permutation $t$-test were performed using QIIME scripts, all other analyses were performed using R 3.2.0 (R Core Team, 2015). Bar plots were created by using GraphPad Prism 6.0.

\section{Data Deposition}

The sequences reported in this study are deposited on the 'European Nucleotide Archive' under the accession number PRJEB2942 $1^{1}$.

\section{RESULTS AND DISCUSSION}

\section{S Sequencing for GM Structure Evaluation of ASD Young Children}

We attempted to identify the key features of GM in very young children (2-4 years of age) at first diagnosis of ASD. High-throughput sequencing analysis of bacterial 16S rRNA V3-V4 regions was conducted on fecal samples of ASD and HCs. In total, over 3.078 million of high-quality sequences $(123,138.64 \pm 47,651.53$ reads/sample) were obtained from all 25 fecal samples, representing 4,870 operational taxonomic units (OTUs). We used a depth of 45,038 sequences/sample clustered in 4,129 OTUs. Good's coverage > of 99\% for all sequences in the two groups suggested that the majority of the phylotypes present in the samples had been identified, indicating good sequencing depth for investigation of ASD associated fecal microbiota (Table 2). Bacterial diversity within communities was significantly higher in the ASD group than in the controls, as indicated by the Shannon index, while no significant differences in number of observed species were detected between the two groups (Table 2). To date, conflicting data are available on the microbial richness of GM in patients with ASD, probably for the diverse enrollment criteria adopted worldwide and age dissimilarity of the studies participants. Therefore, the different bacterial diversity here observed in the

\footnotetext{
${ }^{1}$ https://www.ebi.ac.uk/ena/data/view/PRJEB29421
} 
TABLE 2 | Observed diversity and estimated phylotype coverage for 16S rRNA gene sequences at 97\% similarity from NGS analysis.

\begin{tabular}{|c|c|c|c|c|c|c|c|c|}
\hline \multicolumn{5}{|c|}{ Alpha diversity } & \multicolumn{4}{|c|}{ Beta diversity } \\
\hline Group & $\begin{array}{l}\text { No. of } \\
\text { reads }\end{array}$ & $\begin{array}{l}\text { Observed } \\
\text { species }\end{array}$ & Shannon & $\begin{array}{l}\text { Good's } \\
\text { coverage }\end{array}$ & & $\begin{array}{c}\text { Student's } \\
\text { t-test }\end{array}$ & ANOSIM & ADONIS \\
\hline ASD patients & 495,418 & $920.5 \pm 120.0$ & $5.79 \pm 0.53^{*}$ & $0.993 \pm 0.001$ & $\begin{array}{c}\text { Unweighted } \\
\text { UniFrac } \\
\text { distances }\end{array}$ & $\begin{array}{l}t=-3.001 \\
(p=0.003)\end{array}$ & $\begin{array}{l}R=0.199 \\
(p=0.006)\end{array}$ & $\begin{array}{l}R^{2}=0.074 \\
(p<0.001)\end{array}$ \\
\hline $\mathrm{HCs}$ & 630,532 & $789.2 \pm 255.5$ & $4.73 \pm 1.07$ & $0.994 \pm 0.002$ & $\begin{array}{l}\text { Weighted } \\
\text { UniFrac } \\
\text { distances }\end{array}$ & $\begin{array}{c}t=-6.076 \\
(p=3.76 E-09)\end{array}$ & $\begin{array}{l}R=0.368 \\
(p=0.001)\end{array}$ & $\begin{array}{l}R^{2}=0.246 \\
(p<0.001)\end{array}$ \\
\hline
\end{tabular}

Alpha diversity indexes are reported as mean $\pm S D$; *values are significantly different at $p<0.05$.

two groups might be a distinctive factor in studies involving toddlers/preschoolers.

To evaluate differences in phylogenetic diversity among samples and between groups, beta diversity analysis was conducted by means of unweighted and weighted UniFrac distance metrics. Both measures, represented in two-dimentional principal coordinate analysis ( $\mathrm{PCoA}$ ) plots, showed a clustering of ASD away from the control samples (Figures 1A,B). In particular, the three different statistical methods used to compare beta diversity results showed that a more marked difference was estimated by considering weighted analysis of UniFrac distances. Results indicated that the differences in ASD and HCs microbial communities were prevalently due to changes in OTUs relative abundances (Table 2 and Figures 1A,B). Since two out of 11 patients were female and 2 patients (one male and one female) had symptoms of constipation, we evaluated the impact of these potential confounders on the differences in GM composition between the two groups. Applying MiRKAT to the weighted and unweighted UniFrac distances and adjusting for the small-sample size of the covariates, the described differences in GM composition between ASD and HCs were further confirmed $(p<0.01$ for both weighted and unweighted UniFrac distances after gender and constipation covariate adjustments; Supplementary Figure S1).

\section{Phylogenetic Shift in GM of ASD Patients}

At phylum level, ASD group was characterized by a marked reduction of Actinobacteria $(12.18 \%$ vs. $47.30 \%$ in ASD and HCs, respectively; $p=0.004)$, and a significant increase in Bacteroidetes $(19.34 \%$ vs. $1.53 \%$ in ASD and $\mathrm{HCs}$, respectively; $p=0.04$ ) and Proteobacteria $(9.27 \%$ vs. $0.55 \%$ in ASD and HCs, respectively; $p=0.004$; Figure 2A). Firmicutes represented the most abundant phylum both in HCs and ASD patients, without showing significant differences between the two groups. Bacteroidetes/Firmicutes ratio was significantly higher in ASD patients due to an increase in Bacteroidetes. At family level Actinomycetaceae, Coriobacteriaceae, Bifidobacteriaceae, Gemellaceae and Streptococcaceae were significantly reduced in ASD group (Figure 2B).

Then we identified specific phylotypes marking the differences in GM composition between the ASD and HCs groups. For 16S rRNA gene analyses a very conservative approach was applied

\section{A}

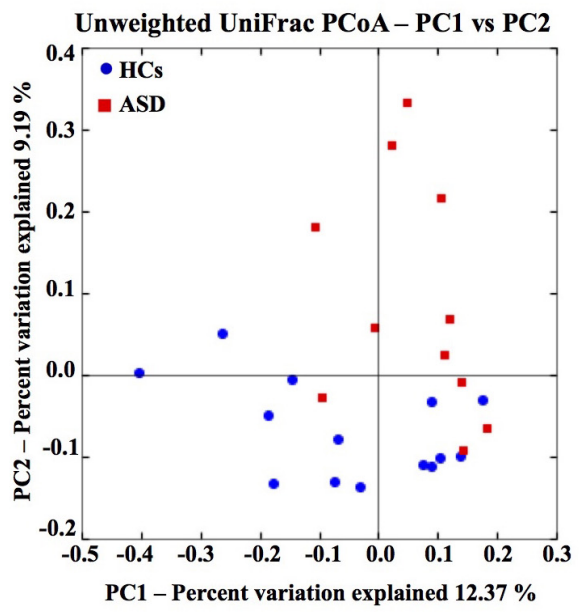

B

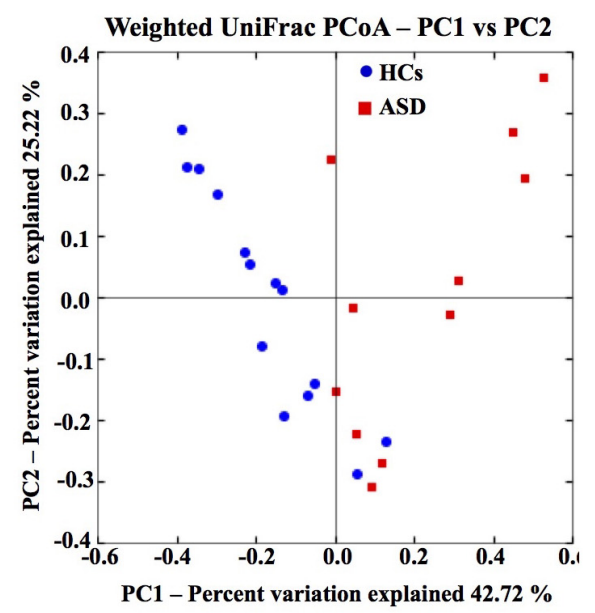

FIGURE 1 | Gut microbiota structure of HCs and ASD patients. Unweighted (A) and weighted (B) UniFrac-based PCoA plot based on all OTUs of gut microbial communities (45,038 sequences/sample). 

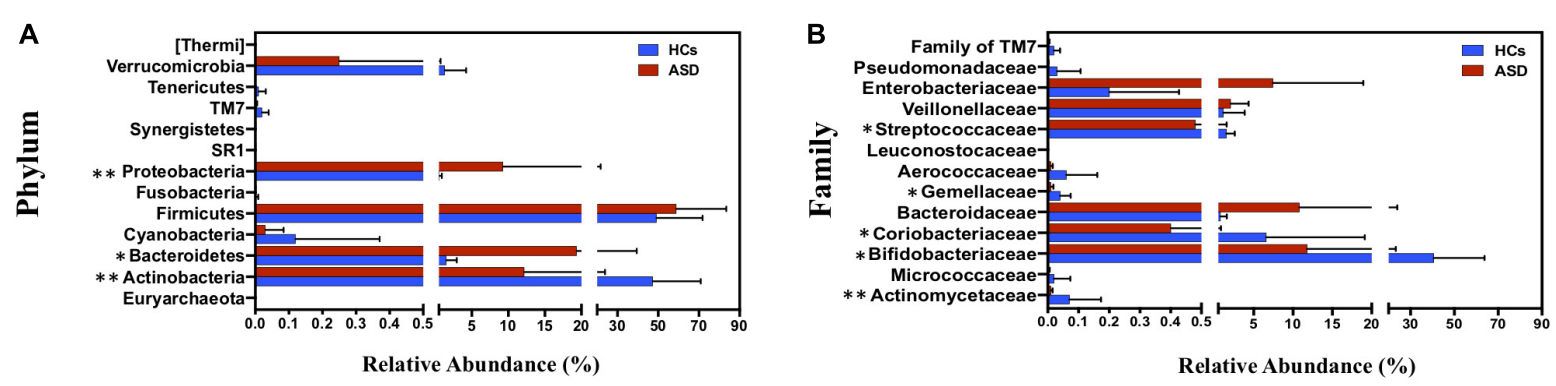

FIGURE 2 | Taxonomic differences of gut microbiota between HCs and ASD groups. (A) Percentage distribution of all bacterial phyla identified and (B) percentage distribution of significant bacterial families $(p<0.05)$ between HCs and ASD. ${ }^{*} p<0.05$, ${ }^{* *} p<0.01$ after FDR correction. Mean values \pm SD are plotted.

using LefSe algorithm and Metastats comparison to detect key OTUs responsible for the differences between ASD and HCs groups (White et al., 2009; Segata et al., 2011). Among total 4,129 OTUs, we found 91 key OTUs (relative abundance $>0.01 \%$ ) defining the GM of ASD patients; specifically, 65/91 OTUs were found more abundant and 26/91 OTUs less abundant in ASD patients with respect to controls (Table 3). To obtain bacterial species assignment of key OTUs, sequencing data were reprocessed using SPINGO high-resolution approach (Allard et al., 2015). This analysis resolved at species level the taxonomic classification of the 91 key OTUs identifying 40 distinct bacterial species (Figure 3).

\section{Low Abundance of Specific Actinobacteria Phylum Components}

Among Actinobacteria, ASD patients showed a significant decrease of OTUs assigned to unclassified genus of Coriobacteriaceae, Actinomyces, Corynebacterium and of OTUs corresponding to Bifidobacterium longum and Eggerthella lenta (Table 3 and Figure 3). Notably, members of Actinobacteria, in particular Bifidobacterium spp., form a dominant fraction of the human GM, particularly in infants (Rajilić-Stojanović and de Vos, 2014). In accordance with our results, several studies reported decrease of Bifidobacteria in ASD patients (Adams et al., 2011; Wang et al., 2011; De Angelis et al., 2013;

TABLE 3 | Greengenes taxonomic classification and relative abundance of key OTUs defining the GM differences between ASD and HCs.

\begin{tabular}{|c|c|c|c|c|c|}
\hline Phylum & Family & Genus & $\begin{array}{c}\text { Number of } \\
\text { OTUs }\end{array}$ & ASD & HCs \\
\hline \multirow[t]{5}{*}{ Actinobacteria } & Actinomycetaceae & Actinomyces & 1 & $0.002 \pm 0.001$ & $0.039 \pm 0.02$ \\
\hline & Corynebacteriaceae & Corynebacterium & 1 & $0.001 \pm 0.001$ & $0.021 \pm 0.007$ \\
\hline & Bifidobacteriaceae & Bifidobacterium (longum) & 1 & $6.904 \pm 2.021$ & $15.302 \pm 2.943$ \\
\hline & Coriobacteriaceae & - & 1 & 0 & $0.026 \pm 0.016$ \\
\hline & Coriobacteriaceae & Eggerthella (lenta) & 1 & $0.081 \pm 0.031$ & $0.408 \pm 0.113$ \\
\hline \multirow[t]{2}{*}{ Bacteroidetes } & Porphyromonadaceae & Parabacteroides (distasonis) & 1 & $0.043 \pm 0.028$ & 0 \\
\hline & Bacteroidaceae & Bacteroides & 22 & $1.606 \pm 1.121$ & $0.003 \pm 0.002$ \\
\hline \multirow[t]{13}{*}{ Firmicutes } & Aerococcaceae & - & 1 & $0.006 \pm 0.002$ & $0.059 \pm 0.027$ \\
\hline & Streptococcaceae & Streptococcus & 5 & $0.065 \pm 0.03$ & $0.704 \pm 0.176$ \\
\hline & U. Clostridiales & - & 3 & $0.09 \pm 0.05$ & $0.1 \pm 0.07$ \\
\hline & & - & 2 & $0.16 \pm 0.15$ & 0 \\
\hline & & Ruminococcus & 3 & $0.42 \pm 0.19$ & $0.04 \pm 0.02$ \\
\hline & Lachnospiraceae & Blautia & 10 & $1.06 \pm 0.38$ & $2.99 \pm 0.55$ \\
\hline & & Coprococcus & 4 & $0.06 \pm 0.02$ & $0.3 \pm 0.08$ \\
\hline & & Lachnospira & 1 & $0.04 \pm 0.03$ & 0 \\
\hline & & Roseburia & 1 & $0.06 \pm 0.05$ & 0 \\
\hline & Peptostreptococcaceae & - & 1 & $0.02 \pm 0.02$ & 0 \\
\hline & & - & 18 & $0.97 \pm 0.36$ & $0.1 \pm 0.02$ \\
\hline & Ruminococcaceae & Faecalibacterium (prausnitzii) & 6 & $0.51 \pm 0.31$ & $0.04 \pm 0.01$ \\
\hline & & Oscillospira & 3 & $1.21 \pm 0.5$ & $0.15 \pm 0.05$ \\
\hline \multirow[t]{2}{*}{ Proteobacteria } & Enterobacteriaceae & - & 4 & $0.407 \pm 0.368$ & $0.002 \pm 0.002$ \\
\hline & Pasteurellaceae & - & 1 & $0.057 \pm 0.049$ & 0 \\
\hline
\end{tabular}

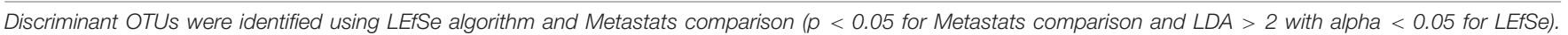

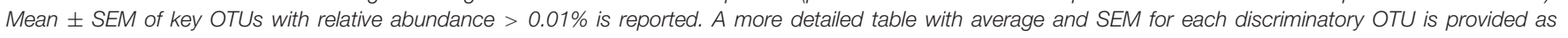
Supplementary Table S1. 


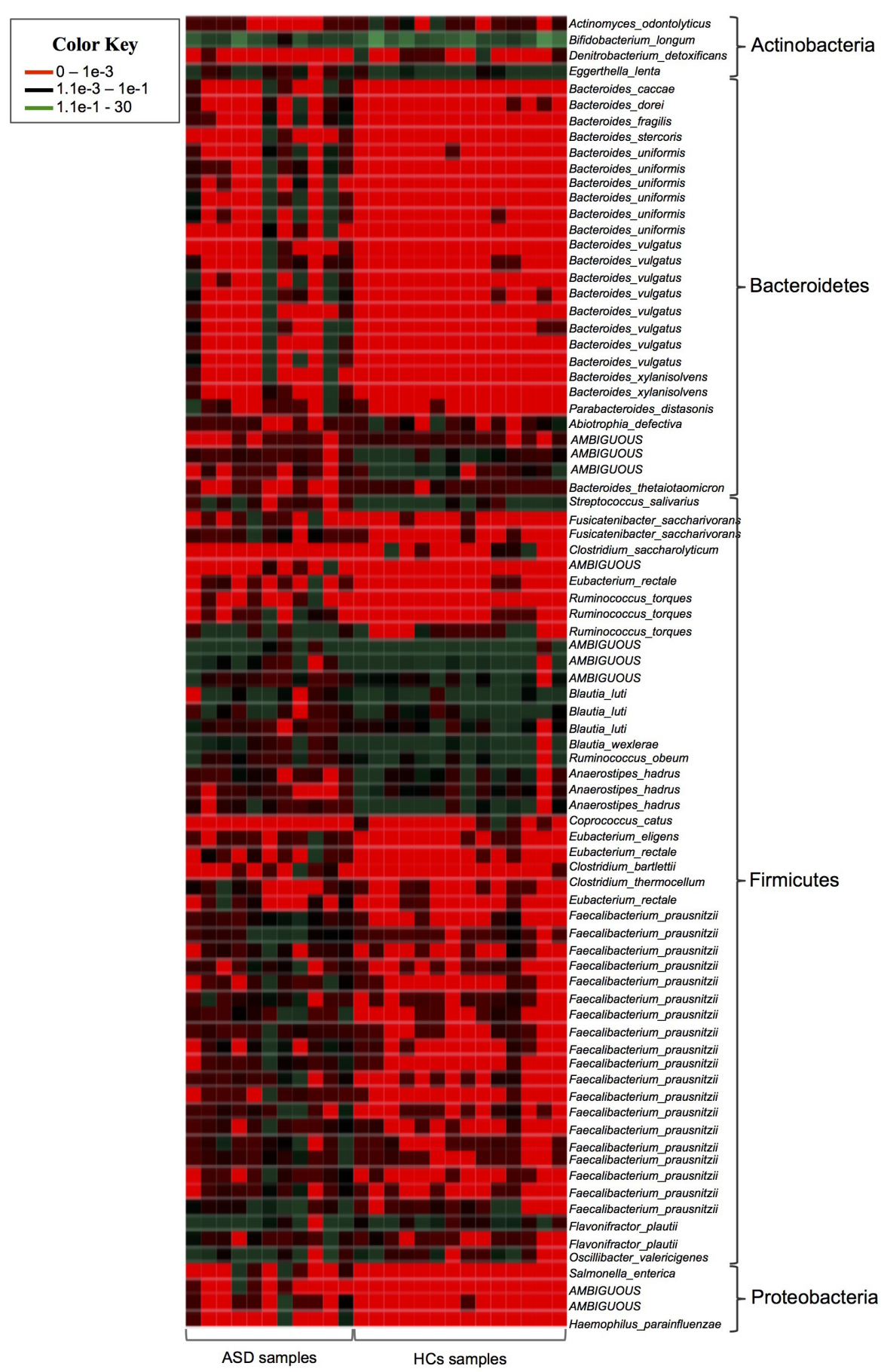

FIGURE 3 | Heatmap showing the SPINGO species classification of the key OTUs with a relative abundance $>0.01 \%$ ( $y$-axis) for individual fecal samples ( $\mathrm{x}$-axis).

Kang et al., 2013, 2017). Bifidobacterium is known as a promoter of healthy status and, recently, it has been proposed as a "psycobiotic" for its ability to produce neuromodulators and influence gut-brain relationship through interaction with other commensal bacteria (Sarkar et al., 2016). The administration of B. longum in animal models improves anxiety, depression, and memory related behaviors (Bercik et al., 2011). In a clinical study a mixture of probiotics including B. longum alleviated psychological distress and urinary cortisol levels (Messaoudi et al., 2011). The severe reduction of B. longum in ASD young children here reported, indicates a re-assortment of key microbes of infants GM in these patients, possibly contributing to some ASD symptoms by affecting gut ecosystem and functionality. 


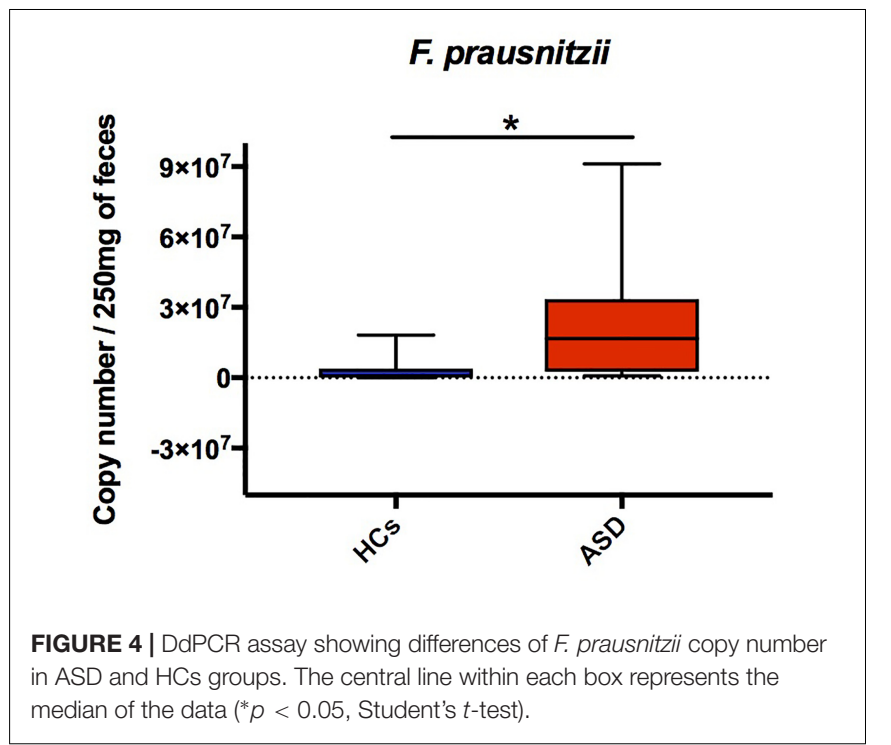

\section{Increase in Members of Gram-Negative Bacterial Phyla}

Our data also showed an increase of several gram-negative bacteria with potential pathogenic features in GM of young children with ASD. Lipopolysaccharide (LPS), the major component of gram-negative cell wall, has been found increased in the serum of ASD patients and was associated with impaired social behavioral scores (Emanuele et al., 2010). LPS stimulates the secretion of proinflammatory cytokines from peripheral blood mononuclear cells and lymphoblasts of ASD children (Jyonouchi et al., 2001), probably contributing to both peripheral and brain inflammation associated with the disease (Patterson, 2011; Depino, 2013; Onore et al., 2013). In our study, among gram-negative bacteria, 22 Bacteroidetes OTUs owing to genus Bacteroides, mainly assigned to B. uniformis and B. vulgatus and $P$. distasonis species, were more abundant in ASD patients as well as Enterobacteriaceae and Pasteurellaceae OTUs belonging to Proteobacteria phylum (Table 3 and Figure 3).

\section{Reassortment of Firmicutes Phylum Components}

The majority of key phylotypes were taxonomically classified in Firmicutes phylum (58/91 OTUs; Table 3 and
Supplementary Table S1 and Figure 3), depicting that, changes within Firmicutes taxa typically characterized the GM of ASD patients. Streptococcus OTUs were markedly reduced in ASD patients and Clostridium clusters were differently represented in ASD and control groups (Table 3). Notably, Clostridium cluster IV discriminatory OTUs assigned to unclassified genus of Ruminococcaceae, Faecalibacterium prausnitzii and Oscillospira were all significantly increased in ASD patients. SPINGO analysis showed that 13/18 OTUs of unclassified genus of Ruminococcaceae were taxonomically classified as F. prausnitzii, revealing that increase in relative abundance of F. prausnitzii marked the GM of ASD children (Figure 3). GM studies involving ASD patients have shown inconsistent results regarding the abundance of $F$. prausnitzii (Wang et al., 2011; De Angelis et al., 2013; Kang et al., 2013, 2017). These conflicting data might depend on several factors ranging from the sampling cohort to the techniques and software used to identify the GM composition. To complement and support our findings, the copy number of $F$. prausnitzii was accurately quantified using Droplet digital PCR (ddPCR) as adjunctive approach to microbiota sequencing. The analysis revealed that $F$. prausnitzii was numerically more abundant in ASD patients compared to HCs (Figure 4). The ddPCR assay also showed no significant differences in number of total bacteria in fecal samples between ASD patients and controls (data not shown). Differently from Bifidobacteria, F. prausnitzii is a late GM colonizer in healthy subjects and it is present at very low levels until childhood (Miquel et al., 2014). The high levels of $F$. prausnitzii in ASD patients indicate its gut premature colonization possibly at the expense of other beneficial bacteria such as B. longum.

\section{Evaluation of Fecal SCFAs Levels and Analysis of KEGG Functions Related to Butyrate Production and Mucin Degradation in ASD Patients}

The higher amount of Bacteroidetes together with the altered assortment in Firmicutes taxa in ASD patients can impact on fecal SCFAs levels. SCFAs are the main end-products of bacterial fermentation in the gut and are among the most important components of microbe-host signaling, able to modulate gene
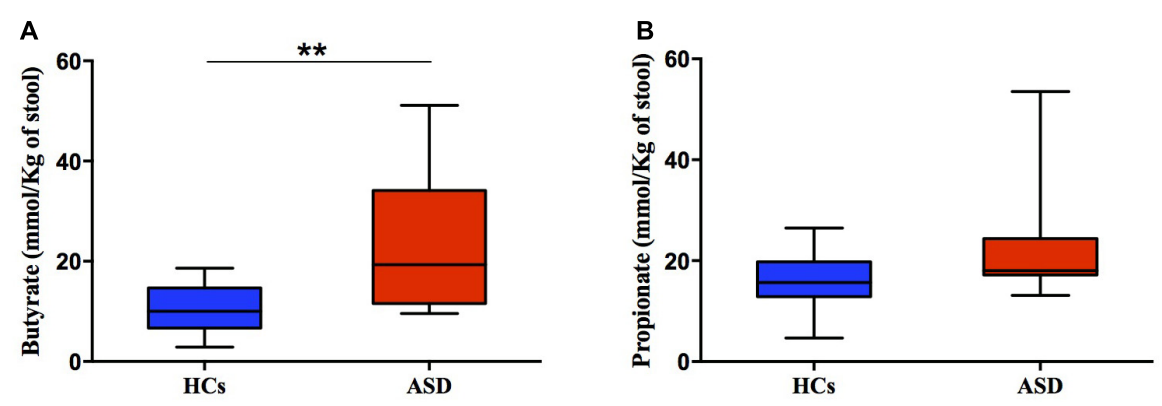

FIGURE 5 | Fecal concentration (mmol/Kg) of butyric (A) and propionic acid (B) in ASD and HCs samples. ${ }^{* *} p<0.01$ for comparisons of ASD vs. HCs using Mann-Whitney test. 
TABLE 4 | PICRUSt KEGG Ortholog count prediction of genes codifying for key enzymes involved in butyrate production in ASD and HCs samples.

\begin{tabular}{|c|c|c|}
\hline KEGG ortholog: description & ASD & HCs \\
\hline $\begin{array}{l}\text { K00626: acetyl-CoA } \\
\text { C-acetyltransferase }\end{array}$ & $11104 \pm 1470.89$ & $7656.86 \pm 1303.27$ \\
\hline $\begin{array}{l}\text { K00074: 3-hydroxybutyryl-CoA } \\
\text { dehydrogenase] }\end{array}$ & $11688.64 \pm 1977.89$ & $12879.64 \pm 1142.51$ \\
\hline K01692: enoyl-CoA hydratase & $2083.91 \pm 486.13$ & $1547.21 \pm 232.23$ \\
\hline $\begin{array}{l}\text { K01715: 3-hydroxybutyryl-CoA } \\
\text { dehydratase }\end{array}$ & $7977.64 \pm 2070.13$ & $6119.93 \pm 1372$ \\
\hline $\begin{array}{l}\text { K00248: butyryl-CoA } \\
\text { dehydrogenase }\end{array}$ & $11984.45 \pm 2138.89$ & $8469.43 \pm 1746.3$ \\
\hline $\begin{array}{l}\text { K00634: phosphate } \\
\text { butyryltransferase }\end{array}$ & $2770.27 \pm 621.97^{*}$ & $505.71 \pm 99.14$ \\
\hline K00929: butyrate kinase & $6114.18 \pm 867.86$ & $3918.14 \pm 883.67$ \\
\hline $\begin{array}{l}\text { K01034: acetate } \\
\text { CoA-transferase alpha subunit }\end{array}$ & $649.36 \pm 167.35^{*}$ & $148.07 \pm 44.15$ \\
\hline $\begin{array}{l}\text { K00016: L-lactate } \\
\text { dehydrogenase }\end{array}$ & $17782.91 \pm 3128.77$ & $24697.43 \pm 2038.36$ \\
\hline
\end{tabular}

Data are mean \pm SEM. Significant differences ( $\left.{ }^{*} p<0.05\right)$ were assessed using nonparametric Kruskal-Wallis test taking into account False Discovery Rate (FDR) corrected p-values.

expression in host cells, brain function and behavior, host energy metabolism and immune functions (Kim et al., 2016; Russo et al., 2016).

Here, analysis of fecal SCFAs levels, namely butyrate and propionate, revealed a trend toward an increase in ASD patients (Figures 5A,B). Butyrate and propionate fecal levels resulted within the normal range in all study subjects. But butyrate levels, even though remaining in normal limits, were significantly higher in ASD (median $=19.30 \mathrm{mmol} / \mathrm{kg}$ ) compared to HCs group $($ median $=10.00 \mathrm{mmol} / \mathrm{kg}, p=0.005 ;$ Figure 5A). Results suggest differences in the colonic fermentation in the two groups. In particular, data show that marked increase of $F$. prausnitzii, one of the most human butyrogenic bacteria, could account for augment of fiber fermentation capability and higher butyrate levels from gut microbes in ASD children.

PICRUSt analysis was used to predict and analyze the KEGG functions involved in butyrate production (Table 4; Langille et al., 2013; Borrelli et al., 2017). Notably, the majority of the analyzed functions were enriched in ASD patients compared to controls. In particular, phosphate butyryltransferase (K00634) and acetate CoA-transferase alpha subunit (K01034), enzymatic functions involved in the final conversion of butyryl-CoA in butyrate, were both significantly higher in ASD microbial communities (Table 4). Butyrate can also be produced from mucin degradation, thus we searched for the counts of beta-hexosaminidases, a mucin-degrading enzyme. We found beta-hexosaminidase (K12373) significantly more abundant in ASD group $(13,815.45 \pm 3,547.44$ and $3,582.57 \pm 564.94$ in ASD and HCs, respectively, $p<0.05$ after FDR correction); we also observed that high abundance of the mucin-degrading enzyme in ASD patients was concurrent with the increased level of Ruminococcus torques which is known to be a mucin-degrading bacterium (Figure 3). Crossfeeding mechanisms in gut ecosystem have been defined to connect metabolic activity of lactic acid-producing and mucin degrading bacteria (e.g., Bifidobacterium and Ruminococcus, respectively) with butyrate producing pathways (Duncan et al., 2004; Schwab et al., 2014). Our data pinpoint the possibility that the reduction of Bifidobacteria could be counterweighed by increase of mucin degrading bacteria to sustain augment of butyrogenic F. prausnitzii in ASD young children. Our observations were also sustained by results obtained from co-occurrence and exclusion network analysis based on Spearman's correlation coefficients, among microbial community structure of ASD patients and HCs, autism indices (ADOS score) and fecal butyrate levels (Figure 6). Co-occurrence network analysis revealed three ASD-enriched OTUs belonging to F. prausnitzii, B. uniformis, and B. vulgatus

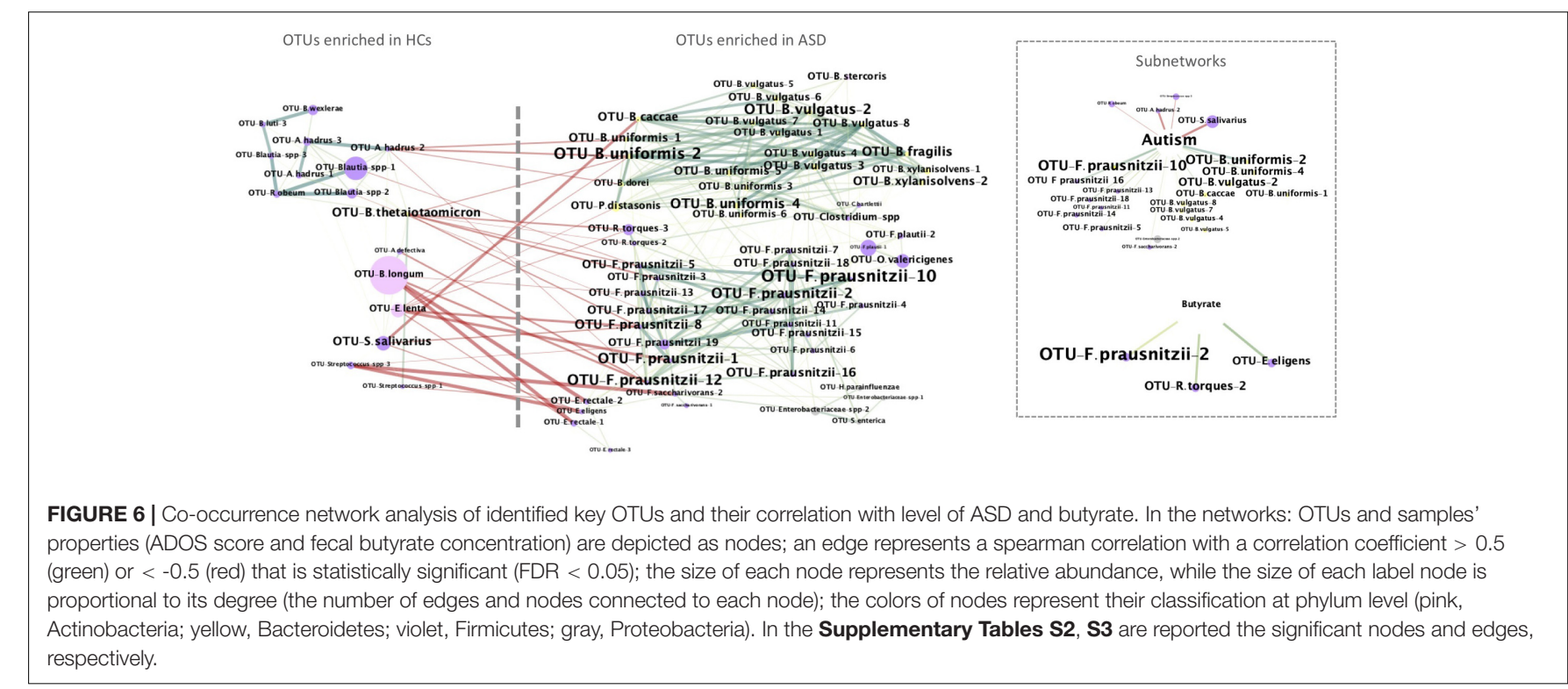


species as highly interconnected and potentially able to drive, in the gut of ASD patients, the presence of other 35 OTUs and generate high bacterial co-occurrence network complexity. The three mentioned ASD-enriched OTUs were also the phylotypes most negatively correlated with HCs-enriched OTUs annotated to B. longum, E. lenta, and Streptococcus OTUs, suggesting their antagonistic or mutual exclusion relationship (Figure 6). The analysis also showed few OTUs directly associated with the level of ASD and concentrations of fecal butyrate. Notably, most ASD-enriched phylotypes, such as $F$. prausnitzii and $B$. uniformis, were positively correlated to the ADOS score $(R=0.767$ and $R=0.84$ with $p<0.001$ for correlation with $F$. prausnitzii and B. uniformis, respectively), to which HCs-enriched phylotypes were negatively correlated (Figure 6, sub-networks). Finally, 3 OTUs taxonomically classified as F. prausnitzii, R. torques, and E. eligens, were found positively associated to butyrate levels (Figure 6, sub-networks).

Overall we observed a reassortment of gut ecosystem in young ASD patients as off early childhood characterized by shift in colonization of gut beneficial bacterial species. The majority of patients enrolled did not suffer from GI symptoms or comorbidities, thus the observed changes in microbial colonization depended primarily on autistic disorder itself. We reported a strong depletion of $B$. longum, with Bifidobacterium species defined as psicobiotics (Sarkar et al., 2016), preservers of gut barrier function and immune homeostasis (Sarkar et al., 2016). Concurrently, in accordance with a previous study (Wang et al., 2013), we also detected increased levels of the mucin-degrading $R$. torques in feces of ASD children. Mucus degradation might affect intestinal epithelium permeability contributing to the development of leaky gut phenotype also described for ASD children (Li et al., 2017). Moreover, we detected significant higher levels of butyrogenic F. prausnitzii and butyrate in feces of ASD patients with respect to controls. Other authors also reported overrepresentation of butyrate producing bacteria in patients with major depressive disorders (Zheng et al., 2016). Butyrate has beneficial effects on human gut homeostasis (Berni Canani et al., 2011; Macia et al., 2015), host immune functions (Kim et al., 2016) and is considered a resourceful compound with promising effects in several neurologic and neuropsichiatric disorders (Bourassa et al., 2016; Stilling et al., 2016; Dinan and Cryan, 2017). Preliminary data from animal models suggested a dose-dependency of butyrate effects on the brain development and function. With positive effects on brain functions and behavior of low doses of butyrate $(<500 \mathrm{mg} / \mathrm{kg})$ and stress-like response elicited by high doses of butyrate $(>1,000 \mathrm{mg} / \mathrm{kg}$ ) (Gagliano et al., 2014; Takuma et al., 2014; Kratsman et al., 2016). The butyrate fecal concentration observed in ASD children in our study was within the normal range and significantly lower compared with the butyrate concentration potentially responsible for brain stress. This data strongly limits the hypothesis of a pathogenetic action elicited by butyrate in ASD. We are conscious that variations of fecal SCFAs levels in feces could depend on either poor absorption due to increased gut permeability or excessive colonic fermentation, or different exposure to environmental factors. Our data suggest that both, microbial composition (namely increase of $R$. torques and $F$. prausnitzii) and microbial predicted metabolic activity (namely increase in mucin degradation and butyrate formation enzymes) may be accountable for the observed higher level of butyrate in fecal content of ASD patients.

This study, despite limitations relative to the small number of children evaluated, encourage to further investigate the role of GM-brain axis in the critical neurodevelopmental window of early life.

\section{CONCLUSION}

Our findings sustain the global alteration of GM equilibrium in ASD young children, including abnormalities in temporal colonization by B. longum and F. prausnitzii. The parallel development between GM and brain circuits, especially those required for social and emotional cognition, strongly suggest a role for GM and their metabolites in ASD symptoms and progress. In this view, it is very important to identify age-related bacterial signatures in ASD in order to develop GM-based therapies.

\section{AUTHOR CONTRIBUTIONS}

LCo performed microbiota analysis, statistically analyzed all data and wrote the manuscript. LP produced and analyzed butyrate and propionate data and contributed to manuscript writing. $\mathrm{MR}$ and GCo organized the clinical work and performed the subject's enrolment. FA, MCo, and GCa performed ddPCR and revised the manuscript. $\mathrm{CD}$ produced butyrate and propionate data. $\mathrm{MCu}, \mathrm{AN}, \mathrm{LB}$, and $\mathrm{EB}$ contributed to the metagenomic analysis and to the interpretation of the data. LCh conceived the investigation and contributed to the interpretation of the data. $\mathrm{CB}$ designed the epidemiological study, organized the clinical work, and performed the subject's enrolment. RB conceived the investigation, designed the epidemiological study, supervised the general aspects of the work and contributed to manuscript writing. FL conceived the investigation, interpreted the data, wrote the manuscript, and critically supervised the work. All authors reviewed the manuscript.

\section{ACKNOWLEDGMENTS}

We would like to thank Domenico Palumbo and Ornella Affinito for help in statistical analysis.

\section{SUPPLEMENTARY MATERIAL}

The Supplementary Material for this article can be found online at: https://www.frontiersin.org/articles/10.3389/fmicb. 2018.03146/full\#supplementary-material 


\section{REFERENCES}

Adams, J. B., Johansen, L. J., Powell, L. D., Quig, D., and Rubin, R. A. (2011). Gastrointestinal flora and gastrointestinal status in children with autismcomparisons to typical children and correlation with autism severity. BMC Gastroenterol. 11:22. doi: 10.1186/1471-230X-11-22

Allard, G., Ryan, F. J., Jeffery, I. B., and Claesson, M. J. (2015). SPINGO: a rapid species-classifier for microbial amplicon sequences. BMC Bioinformatics 16:324. doi: 10.1186/s12859-015-0747-1

American Psychiatric Association. (2013). Cautionary Statement for Forensic Use of DSM-5. In Diagnostic and Statistical Manual of Mental Disorders, 5th Edn. Washington, DC: American Psychiatric Association Publishing, 280. doi: 10. 1176/appi.books.9780890425596.744053

Bercik, P., Park, A. J., Sinclair, D., Khoshdel, A., Lu, J., Huang, X., et al. (2011). The anxiolytic effect of Bifidobacterium longum NCC3001 involves vagal pathways for gut-brain communication. Neurogastroenterol. Motil. 23, 1132-1139. doi: $10.1111 / \mathrm{j} .1365-2982.2011 .01796 . \mathrm{x}$

Berni Canani, R., Costanzo, M. D., Leone, L., Pedata, M., Meli, R., and Calignano, A. (2011). Potential beneficial effects of butyrate in intestinal and extraintestinal diseases. World J. Gastroenterol. 17, 1519-1528. doi: 10.3748/ wjg.v17.i12

Borrelli, L., Coretti, L., Dipineto, L., Bovera, F., Menna, F., Chiariotti, L., et al. (2017). Insect-based diet, a promising nutritional source, modulates gut microbiota composition and SCFAs production in laying hens. Sci. Rep. 7:16269. doi: 10.1038/s41598-017-16560-6

Bourassa, M. W., Alim, I., Bultman, S. J., and Ratan, R. R. (2016). Butyrate, neuroepigenetics and the gut microbiome: can a high fiber diet improve brain health? Neurosci. Lett. 625, 56-63. doi: 10.1016/j.neulet.2016. 02.009

Caporaso, J. G., Kuczynski, J., Stombaugh, J., Bittinger, K., Bushman, F. D., Costello, E. K., et al. (2010). QIIME allows analysis of high-throughput community sequencing data. Nat. Methods 7, 335-336. doi: 10.1038/nmeth. f.303

Chen, Y., Yang, F., Lu, H., Wang, B., Chen, Y., Lei, D., et al. (2011). Characterization of fecal microbial communities in patients with liver cirrhosis. Hepatology 54, 562-572. doi: 10.1002/hep.24423

Core Team, R. (2015). R: A Language and Environment for Statistical Computing. Vienna: R Found. Stat. Comput.

Coretti, L., Cristiano, C., Florio, E., Scala, G., Lama, A., Keller, S., et al. (2017). Sexrelated alterations of gut microbiota composition in the BTBR mouse model of autism spectrum disorder. Sci. Rep. 7:45356. doi: 10.1038/srep45356

De Angelis, M., Piccolo, M., Vannini, L., Siragusa, S., De Giacomo, A., Serrazzanetti, D. I., et al. (2013). Fecal microbiota and metabolome of children with autism and pervasive developmental disorder not otherwise specified. PLoS One 8:e76993. doi: 10.1371/journal.pone.0076993

de Magistris, L., Familiari, V., Pascotto, A., Sapone, A., Frolli, A., Iardino, P., et al. (2010). Alterations of the intestinal barrier in patients with autism spectrum disorders and in their first-degree relatives. J. Pediatr. Gastroenterol. Nutr. 51, 418-424. doi: 10.1097/MPG.0b013e3181dcc4a5

de Theije, C. G. M., Wopereis, H., Ramadan, M., van Eijndthoven, T., Lambert, J., Knol, J., et al. (2014). Altered gut microbiota and activity in a murine model of autism spectrum disorders. Brain Behav. Immun. 37, 197-206. doi: 10.1016/j. bbi.2013.12.005

Depino, A. M. (2013). Peripheral and central inflammation in autism spectrum disorders. Mol. Cell. Neurosci. 53, 69-76. doi: 10.1016/j.mcn.2012.10.003

DeSantis, T. Z., Hugenholtz, P., Larsen, N., Rojas, M., Brodie, E. L., Keller, K., et al. (2006). Greengenes, a chimera-checked 16S rRNA gene database and workbench compatible with ARB. Appl. Environ. Microbiol. 72, 5069-5072. doi: 10.1128/AEM.03006-05

Dinan, T. G., and Cryan, J. F. (2017). The microbiome-gut-brain axis in health and disease. Gastroenterol. Clin. North Am. 46, 77-89. doi: 10.1016/j.gtc.2016. 09.007

Duncan, S. H., Louis, P., and Flint, H. J. (2004). Lactate-utilizing bacteria, isolated from human feces, that produce butyrate as a major fermentation product. Appl. Environ. Microbiol. 70, 5810-5817.

Emanuele, E., Orsi, P., Boso, M., Broglia, D., Brondino, N., Barale, F., et al. (2010). Low-grade endotoxemia in patients with severe autism. Neurosci. Lett. 471, 162-165. doi: 10.1016/j.neulet.2010.01.033
Estes, M. L., and McAllister, A. K. (2015). Immune mediators in the brain and peripheral tissues in autism spectrum disorder. Nat. Rev. Neurosci. 16, 469-486. doi: $10.1038 / \mathrm{nrn} 3978$

Faust, K., and Raes, J. (2016). CoNet app: inference of biological association networks using cytoscape. F1000Res. 5:1519.

Finegold, S. M., Dowd, S. E., Gontcharova, V., Liu, C., Henley, K. E., Wolcott, R. D., et al. (2010). Pyrosequencing study of fecal microflora of autistic and control children. Anaerobe 16, 444-453. doi: 10.1016/j.anaerobe.2010.06.008

Frye, R. E., Rose, S., Chacko, J., Wynne, R., Bennuri, S. C., Slattery, J. C., et al. (2016). Modulation of mitochondrial function by the microbiome metabolite propionic acid in autism and control cell lines. Transl. Psychiatry 6:e927. doi: $10.1038 /$ tp. 2016.189

Gagliano, H., Delgado-Morales, R., Sanz-Garcia, A., and Armario, A. (2014). High doses of the histone deacetylase inhibitor sodium butyrate trigger a stresslike response. Neuropharmacology 79, 75-82. doi: 10.1016/j.neuropharm.2013. 10.031

Griffiths, R. (2006). Griffiths Mental Developmental Scales Extended Revised Manual. Firenze: Giunti Organizzazioni Speciali.

Hallmayer, J., Cleveland, S., Torres, A., Phillips, J., Cohen, B., Torigoe, T., et al. (2011). Genetic heritability and shared environmental factors among twin pairs with autism. Arch. Gen. Psychiatry 68, 1095-1102. doi: 10.1001/ archgenpsychiatry.2011.76

Hospodsky, D., Qian, J., Nazaroff, W. W., Yamamoto, N., Bibby, K., RismaniYazdi, H., et al. (2012). Human occupancy as a source of indoor airborne bacteria. PLoS One 7:e34867. doi: 10.1371/journal.pone.0034867

Hsiao, E. Y., McBride, S. W., Hsien, S., Sharon, G., Hyde, E. R., McCue, T., et al. (2013). Microbiota modulate behavioral and physiological abnormalities associated with neurodevelopmental disorders. Cell 155, 1451-1463. doi: 10. 1016/j.cell.2013.11.024

Jyonouchi, H., Sun, S., and Le, H. (2001). Proinflammatory and regulatory cytokine production associated with innate and adaptive immune responses in children with autism spectrum disorders and developmental regression. J. Neuroimmunol. 120, 170-179. doi: 10.1016/S0165-5728(01)00 421-0

Kanehisa, M., Sato, Y., Kawashima, M., Furumichi, M., and Tanabe, M. (2016). KEGG as a reference resource for gene and protein annotation. Nucleic Acids Res. 44, D457-D462. doi: 10.1093/nar/gkv1070

Kang, D. W., Adams, J. B., Gregory, A. C., Borody, T., Chittick, L., Fasano, A., et al. (2017). Microbiota transfer therapy alters gut ecosystem and improves gastrointestinal and autism symptoms: an open-label study. Microbiome 5:10. doi: 10.1186/s40168-016-0225-7

Kang, D. W., Park, J. G., Ilhan, Z. E., Wallstrom, G., LaBaer, J., Adams, J. B., et al. (2013). Reduced incidence of prevotella and other fermenters in intestinal microflora of autistic children. PLoS One 8:e68322. doi: 10.1371/journal.pone. 0068322

Kats, D., Payne, L., Parlier, M., and Piven, J. (2013). Prevalence of selected clinical problems in older adults with autism and intellectual disability. J. Neurodev. Disord. 5, 1-27. doi: 10.1186/1866-1955-5-27

Kim, M., Qie, Y., Park, J., and Kim, C. H. (2016). Gut microbial metabolites fuel host antibody responses. Cell Host Microbe 20, 202-214. doi: 10.1016/j.chom. 2016.07.001

Kratsman, N., Getselter, D., and Elliott, E. (2016). Sodium butyrate attenuates social behavior deficits and modifies the transcription of inhibitory/excitatory genes in the frontal cortex of an autism model. Neuropharmacology 102, 136-145. doi: 10.1016/j.neuropharm.2015.11.003

Langille, M., Zaneveld, J., Caporaso, J. G., McDonald, D., Knights, D., Reyes, J., et al. (2013). Predictive functional profiling of microbial communities using $16 \mathrm{~S}$ rRNA marker gene sequences. Nat. Biotechnol. 31, 814-821. doi: 10.1038/nbt. 2676

Li, Q., Han, Y., Dy, A. B. C., and Hagerman, R. J. (2017). The gut microbiota and autism spectrum disorders. Front. Cell. Neurosci. 11:120. doi: 10.3389/fncel. 2017.00120

Lord, C., Rutter, M., DiLavore, P., Risi, S., and Gotham, K. (2012). Autism Diagnostic Observation Schedule, (ADOS-2). Los Angeles, CA: Western Psychological Services.

MacFabe, D. F., Cain, D. P., Rodriguez-Capote, K., Franklin, A. E., Hoffman, J. E., Boon, F., et al. (2007). Neurobiological effects of intraventricular propionic acid in rats: possible role of short chain fatty acids on the pathogenesis and 
characteristics of autism spectrum disorders. Behav. Brain Res. 176, 149-169. doi: 10.1016/j.bbr.2006.07.025

Macia, L., Tan, J., Vieira, A. T., Leach, K., Stanley, D., Luong, S., et al. (2015). Metabolite-sensing receptors GPR43 and GPR109A facilitate dietary fibre-induced guthomeostasis through regulation of the inflammasome. Nat. Commun. 6:6734. doi: 10.1038/ncomms7734

Messaoudi, M., Violle, N., Bisson, J. F., Desor, D., Javelot, H., and Rougeot, C. (2011). Beneficial psychological effects of a probiotic formulation (Lactobacillus helveticus R0052 and Bifidobacterium longum R0175) in healthy human volunteers. Gut Microbes 2, 256-261. doi: 10.4161/gmic.2.4. 16108

Miquel, S., Martin, R., Bridonneau, C., Robert, V., Sokol, H., Bermúdez-Humarán, L. G., et al. (2014). Ecology and metabolism of the beneficial intestinal commensal bacterium Faecalibacterium prausnitzii. Gut Microbes 5, 146-151. doi: $10.4161 /$ gmic. 27651

Onore, C. E., Careaga, M., Babineau, B. A., Schwartzer, J. J., Berman, R. F., and Ashwood, P. (2013). Inflammatory macrophage phenotype in BTBR $\mathrm{T}+\mathrm{tf} / \mathrm{J}$ mice. Front. Neurosci. 7:158. doi: 10.3389/fnins.2013.00158

Parracho, H. M., Bingham, M. O., Gibson, G. R., and McCartney, A. L. (2005). Differences between the gut microflora of children with autistic spectrum disorders and that of healthy children. J. Med. Microbiol. 54, 987-991. doi: 10.1099/jmm.0.46101-0

Patterson, P. H. (2011). Maternal infection and immune involvement in autism. Trends Mol. Med. 17, 389-394. doi: 10.1016/j.molmed.2011. 03.001

Rajilić-Stojanović, M., and de Vos, W. M. (2014). The first 1000 cultured species of the human gastrointestinal microbiota. FEMS Microbiol. Rev. 38, 996-1047. doi: 10.1111/1574-6976.12075

Raveh-Sadka, T., Thomas, B. C., Singh, A., Firek, B., Brooks, B., Castelle, C. J., et al. (2015). Gut bacteria are rarely shared by co-hospitalized premature infants, regardless of necrotizing enterocolitis development. Elife 4:e05477. doi: 10. 7554/eLife.05477

Russo, R., De Caro, C., Avagliano, C., Cristiano, C., La Rana, G., Mattace Raso, G., et al. (2016). Sodium butyrate and its synthetic amide derivative modulate nociceptive behaviors in mice. Pharmacol. Res. 103, 279-291. doi: 10.1016/j. phrs.2015.11.026

Rutter, M., Le Couteur, A., and Lord, C. (2005). Autism Diagnostic Interview Revised. Firenze: Giunti Organizzazioni Speciali.

Sarkar, A., Lehto, S. M., Harty, S., Dinan, T. G., Cryan, J. F., and Burnet, P. W. J. (2016). Psychobiotics and the manipulation of bacteria-gut-brain signals. Trends Neurosci. 39, 763-781. doi: 10.1016/j.tins.2016.09.002

Schmieder, R., and Edwards, R. (2011). Quality control and preprocessing of metagenomic datasets. Bioinformatics 27, 863-864. doi: 10.1093/ bioinformatics/btr026

Schopler, E., Reichler, R. J., and Renner, B. R. (1988). Childhood Autism Rating Scale (CARS). Los Angeles, CA: West. Psychol. Serv, 59804.

Schwab, C., Berry, D., Rauch, I., Rennisch, I., Ramesmayer, J., Hainzl, E., et al. (2014). Longitudinal study of murine microbiota activity and interactions with the host during acute inflammation and recovery. ISME J. 8, 1101-1114. doi: 10.1038/ismej.2013.223

Segata, N., Izard, J., Waldron, L., Gevers, D., Miropolsky, L., Garrett, W. S., et al. (2011). Metagenomic biomarker discovery and explanation. Genome Biol. 12:R60. doi: 10.1186/gb-2011-12-6-r60

Shannon, P., Markiel, A., Ozier, O., Baliga, N. S., Wang, J. T., Ramage, D., et al. (2003). Cytoscape: a software environment for integrated models of biomolecular interaction networks. Genome Res. 13, 2498-2504.

Song, Y., Liu, C., and Finegold, S. M. (2004). Real-time PCR quantitation of clostridia in feces of autistic children. Appl. Environ. Microbiol. 70, 6459-6465. doi: 10.1128/AEM.70.11.6459-6465.2004

Sparrow, S. E., Balla, D. A., and Cicchetti, D. V. (2003). Vineland Adaptive Behavior Scales. Intervista - Forma Completa. Firenze: Giunti Organizzazioni Speciali.

Stilling, R. M., van de Wouw, M., Clarke, G., Stanton, C., Dinan, T. G., and Cryan, J. F. (2016). The neuropharmacology of butyrate: the bread and butter of the microbiota-gut-brain axis? Neurochem. Int. 99, 110-132. doi: 10.1016/j.neuint. 2016.06.011
Strati, F., Cavalieri, D., Albanese, D., De Felice, C., Donati, C., Hayek, J., et al. (2017). New evidences on the altered gut microbiota in autism spectrum disorders. Microbiome 5:24. doi: 10.1186/s40168-017-0242-1

Takuma, K., Hara, Y., Kataoka, S., Kawanai, T., Maeda, Y., Watanabe, R., et al. (2014). Chronic treatment with valproic acid or sodium butyrate attenuates novel object recognition deficits and hippocampal dendritic spine loss in a mouse model of autism. Pharmacol. Biochem. Behav. 126, 43-49. doi: 10.1016/ j.pbb.2014.08.013

Thomas, R. H., Meeking, M. M., Mepham, J. R., Tichenoff, L., Possmayer, F., Liu, S., et al. (2012). The enteric bacterial metabolite propionic acid alters brain and plasma phospholipid molecular species: further development of a rodent model of autism spectrum disorders. J. Neuroinflammation 9:153. doi: 10.1186/17422094-9-153

Tomova, A., Husarova, V., Lakatosova, S., Bakos, J., Vlkova, B., Babinska, K., et al. (2015). Gastrointestinal microbiota in children with autism in Slovakia. Physiol. Behav. 138, 179-187. doi: 10.1016/j.physbeh.2014.10.033

Walker, L., SCaplan, A., and Rasquin, A. (2006). Rome III Diagnostic Questionnaire for the Pediatric Functional GI Disorders. Rome: The Functional Gastrointestinal Disorders, 961-990.

Wang, L., Christophersen, C. T., Sorich, M. J., Gerber, J. P., Angley, M. T., and Conlon, M. A. (2011). Low relative abundances of the mucolytic bacterium Akkermansia muciniphila and Bifidobacterium spp. in feces of children with autism. Appl. Environ. Microbiol. 77, 6718-6721. doi: 10.1128/AEM.05212-11

Wang, L., Christophersen, C. T., Sorich, M. J., Gerber, J. P., Angley, M. T., and Conlon, M. A. (2012). Elevated fecal short chain fatty acid and ammonia concentrations in children with autism spectrum disorder. Dig. Dis. Sci. 57, 2096-2102. doi: 10.1007/s10620-012-2167-7

Wang, L., Christophersen, C. T., Sorich, M. J., Gerber, J. P., Angley, M. T., and Conlon, M. A. (2013). Increased abundance of Sutterella spp. and Ruminococcus torques in feces of children with autism spectrum disorder. Mol. Autism 4:42. doi: 10.1186/2040-2392-4-42

White, J. R., Nagarajan, N., and Pop, M. (2009). Statistical methods for detecting differentially abundant features in clinical metagenomic samples. PLoS Comput. Biol. 5:e1000352. doi: 10.1371/journal.pcbi.1000352

Williams, B. L., Hornig, M., Buie, T., Bauman, M. L., Cho Paik, M., Wick, I., et al. (2011). Impaired carbohydrate digestion and transport and mucosal dysbiosis in the intestines of children with autism and gastrointestinal disturbances. PLoS One 6:e24585. doi: 10.1371/journal.pone.0024585

Zhang, J., Kobert, K., Flouri, T., and Stamatakis, A. (2014). PEAR: a fast and accurate illumina paired-end reAd mergeR. Bioinformatics 30, 614-620. doi: 10.1093/bioinformatics/btt593

Zhang, M., Ma, W., Zhang, J., He, Y., and Wang, J. (2018). Analysis of gut microbiota profiles and microbe-disease associations in children with autism spectrum disorders in China. Sci. Rep. 18:13981. doi: 10.1038/s41598-01832219-2

Zhao, N., Chen, J., Carroll, I. M., Ringel-Kulka, T., Epstein, M. P., Zhou, H., et al. (2015). Testing in microbiome-profiling studies with MiRKAT, the microbiome regression-based kernel association test. Am. J. Hum. Genet. 96, 797-807.

Zheng, P., Zeng, B., Zhou, C., Liu, M., Fang, Z., Xu, X., et al. (2016). Gut microbiome remodeling induces depressive-like behaviors through a pathway mediated by the host's metabolism. Mol. Psychiatry 21, 786-796. doi: 10.1038/ mp.2016.44

Conflict of Interest Statement: The authors declare that the research was conducted in the absence of any commercial or financial relationships that could be construed as a potential conflict of interest.

Copyright (C) 2018 Coretti, Paparo, Riccio, Amato, Cuomo, Natale, Borrelli, Corrado, De Caro, Comegna, Buommino, Castaldo, Bravaccio, Chiariotti, Berni Canani and Lembo. This is an open-access article distributed under the terms of the Creative Commons Attribution License (CC BY). The use, distribution or reproduction in other forums is permitted, provided the original author(s) and the copyright owner(s) are credited and that the original publication in this journal is cited, in accordance with accepted academic practice. No use, distribution or reproduction is permitted which does not comply with these terms. 